\title{
Gift Giving and the Evolution of Cooperation*
}

\author{
H Lorne Carmichael W Bentley MacLeod ${ }^{\dagger}$
}

First Version August 1992

This version Janaury 1996

Forthcoming International Economic Review.

\begin{abstract}
Gift giving is a practice common to many societies. In an evolutionary model the social custom of giving gifts at the beginning of a relationship can lead to trust and cooperation. The evolutionary approach makes predictions about the character of the goods that can be used as gifts. For example, gift goods may have little use value even at low levels of consumption. Although the gifts themselves are useless, the institution is not.
\end{abstract}

*Submitted August 1994, revised December 1995

†The authors would like to thank the SSHRCC for support, and Ken binmore, Eddie Dekel, David Donaldson, Hideshi Itoh, Akihiko Matsui, Dan Usher, and three anonymous referees for helpful comments. 


\section{Introduction}

Freedom of association brings with it the right to change one's associates. This mobility can lead to better matches, but it may also undermine cooperation within a match. In repeated prisoner's dilemma games, for example, cooperation is possible because an agent cannot escape punishment if she cheats. This paper illustrates how the custom of gift exchange, by imposing costs at the beginning of a relationship, can support cooperation even in markets where cheaters never get caught.

Gift giving customs are widespread, and the practice is often governed by strange and seemingly arcane rules. In the marriage market, for example, a hopeful suitor may buy a conventional gift good, like a box of chocolates. He will remove the price tag, even though the recipient will probably have a good estimate of the cost. He will enclose the gift in wrapping paper, even though he wants his prospective partner to unwrap it almost immediately. Then he will present it when he meets her at the door, before any other meaningful conversation has begun. He would never consider giving money (the most efficient gift), and if he did so he might find the gift rejected and the door closed in his face.

The above is a very conventional account, but variations of it are played out repeatedly in Western society. What could explain practices like this? Asking the people involved (or one's self) is unlikely to give an answer since the actions are part of a social custom. The actors on each side know that their behavior is appropriate for the situation, and no more. But how was the script written? And why was it written in this particular way?

In this paper a social custom is explicitly defined as a behavioral rule or strategy that is taught to each new generation by its parents. The children in most cases follow the rule unreflectively for the rest of their lives, and in turn teach it to their own children ${ }^{2}$. The convention is therefore a replicator, acting much like a gene in a model of biological evolution. We use techniques originally developed in evolutionary biology to generate predictions about the cultural rules that will survive in evolutionary equilibrium.

Social scientists have been familiar with this approach since it was used by Axelrod

\footnotetext{
${ }^{2}$ See Boas (1928) for a discussion of culture and the role of education. In particular his chapter on "Stability of Culture" discusses the way norms of behaviour become automatic and reinforced through learning and imitation. Coleman's (1988) concept of "social capital" is similar.
} 
(1984). In his "evolution of cooperation" model, strategies in a population play a round robin tournament with all of the other strategies present. The game is an infinitely repeated prisoner's dilemma. As the tournament continues the population can be adjusted to reflect the relative success of each of the strategies. Axelrod found the simple rule "tit for tat" was remarkably successful at invading other populations, dominating a diverse group of strategies, and resisting invasion once established. ${ }^{3}$

The economic context in this paper is related, but may be more realistic. In our society matched pairs of agents also play repeated prisoner's dilemmas. However agents are matched with each other in a random fashion and, more important, they can at any time break up a match to look for a new one. This means that "tit for tat", or any other strategy that starts off cooperatively, will not do very well. A population of such strategies will be invaded by "con men", or "parasites", who defect on the first move and then leave to start a new match. "Tit for tat" is too trusting.

A population of strategies that start out with one or more defections can successfully screen out invaders of this kind. "Mistrust of strangers" has evolutionary value. More interesting, however, is our main result that a stable gift giving custom may emerge. Under this convention, an exchange of gifts at the beginning of a new match will break down mistrust and allow cooperation to start immediately. The reason is simple. A parasite in a gift giving society will have to buy a succession of gifts, while incumbent honest types need buy the gift only once. The gift giving custom lowers the relative return to being a parasite.

Since the prisoner's dilemma is symmetric, the gifts in our model must be exchanged given and received - at the beginning of any new match. It follows that the gifts must be privately inefficient - i.e. they must cost more to purchase than they are worth to the receiver. In a market society the custom may therefore specify a particular set of goods to be used as gift goods. The private cost of purchasing the gift will then rise above its use value. Despite this, the institution may be quite efficient at the social level since the extra payments are almost all transferred to the gift makers. Gift giving has this advantage over

\footnotetext{
3 "Tit for tat" is not evolutionarily stable even though it does well in simulations. Also, the strategy "Pavlov" does better in the very long term if mutations occasionally enter and if there is a small possibility of mistakes. See Nowak and Sigmund (1992).
} 
other methods of supporting cooperation. ${ }^{4}$

Gift giving is a widespread and complex institution, and we do not claim that our model explains all of its variations. For example, our model has nothing to do with altruistic giving within families (Becker, 1981) or with reciprocity as studied by Mauss (1990). It is more closely related to Camerer's (1988) model where gifts are used to signal one's expectations about the quality of a match. However, the predictions of our model allow us to distinguish it from these others.

Most of the predictions follow from the simple idea that a custom, if it has survived years of evolutionary competition, must have had a good "immune system" - i.e. it must have been able to fight off attempts to subvert it. This allows us to say a great deal about the goods which should be chosen as gift goods. For example, the best gift goods will have little use value even at very low levels of consumption. They should also depreciate rapidly, or be consumed as a part of the exchange. The wrapping of gifts makes evolutionary sense, as does the removal of price tags, even though the price will be known to the receiver. More generally, the gift giving customs we study will surround only the important, long term relationships in people's lives. The size of the gifts may also be very small relative to the value of the cooperative behavior they support. Other implications are developed throughout the paper.

The first half of the paper is devoted to establishing the existence of evolutionarily stable equilibria in a matching prisoner's dilemma model with communication and gifts. Evolutionary stability in two person repeated games has been studied by Fudenberg and Maskin (1990) and Maskin (1993). The implications of communication for evolutionary stability in normal form games and signalling games is studied by Robson (1990), Matsui (1991), Kim and Sobel (1992), Binmore and Samuelson (1992), Blume, Kim and Sobel (1992) and Schlag (1994). Section 2 of this paper lays out our model and shows that a result from these papers also holds in our context. Since our game has no efficient Nash

\footnotetext{
${ }^{4}$ In a standard economic framework, cooperation can be sustained when there is a surplus to the continuation of a match (MacLeod and Malcomson, 1989). This surplus can be created by unemployment (Shapiro and Stiglitz (1984)), an exchange of hostages (Williamson (1983)), or reputations (Klein and Leffler 1979), but all of these involve some use of resources. Payments to third parties (Carmichael (1983)) avoid this, but create incentives for collusion. These issues are surveyed in Carmichael (1989).
} 
equilibrium, with costless communication it has no evolutionarily stable equilibrium either.

We then go on to show that with gift giving, interpreted here as a costly message, cooperative evolutionarily stable strategies exist. In Section III we study the empirical implications of our explanation for gifts and contrast them with those of other economic models of gift giving.

\section{The Model}

\subsection{Basic Setup and Definitions}

We will study an economy with a continuum of agents indexed by $i \in[0,1]$, with total mass equal to one. Time is discrete. At the end of each period the fraction $(1-\rho)$ of the agents die, and are replaced with new, unmatched agents at the beginning of the next period. For simplicity it is assumed that matched agents die together. There is no unemployment - all players find or continue a match each period.

More formally, the stages in the history of a match can be summarized as follows.

1. Individuals are matched at the beginning of a period. We denote one individual in a match as $i$ and the other as $j$. Neither agent has any knowledge of the other's actions in previous matches.

2. Each agent simultaneously sends a message to her partner. A message consists of a sequence of signals or words taken from the infinite set $M=\{m(1) \ldots m(n) \ldots\}$. Agent $i$ sends the message $M^{i} \subset M$. These messages are costless. An agent may also send a gift to her partner. The cost of the gift can be chosen to be $g^{i}$ dollars, but the gift confers no direct utility on the other person.

3. Each agent observes the message and gift sent by his partner. We assume that messages can contain only a finite number of words. Let the signal or word with the highest index sent by agent $i$ be $m^{i}\left(\ell_{i}\right)$. We assume that this agent observes the signals $m^{j}(1)$ up to $m^{j}\left(\ell_{i}\right)$ but will not be able to observe any higher signals. 
4. Agents then play a standard prisoner's dilemma with actions $a \in\{C, D\}$ and payoffs $\mu\left(a^{i}, a^{j}\right)$ given by:

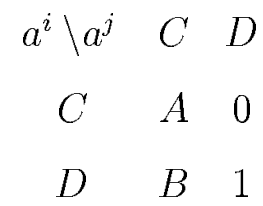

where it is assumed that $2 A>B>A>1$.

5. The payoffs are received (it is now the end of the period).

6. A fraction $(1-\rho)$ of the matches end with the death of each partner. The individuals are replaced with unmatched agents, who follow the same strategies.

7. Agents in the remaining matches simultaneously decide whether to stay for one more period, or to separate. If a separation occurs, then both agents return to the market.

8. The process repeats. New agents and separated agents are randomly and anonymously matched with each other. Matched agents remain with their old partner, but may continue to communicate or exchange gifts if they wish.

Evolution is driven by the entry of new strategies. But if incumbents can accurately identify newcomers, then any strategy that always defects against outsiders will never be invaded. The message technology described in stages 2 and 3 allows agents playing a new strategy to use a "secret handshake" (Robson, 1990) to identify each other while appearing normal to incumbents. This makes it much easier for new strategies to invade. While not completely unrealistic, ${ }^{5}$ this approach is chosen for its simplicity. ${ }^{6}$

The assumption that finite messages come from an infinite message space is consistent with the characteristics of any natural language: with a finite alphabet and grammar there

\footnotetext{
${ }^{5}$ The freemasons are probably the best Western example of a society with secret ways for members to identify each other. Hidishi Itoh has told us that residents of Kyoto have developed a set of customs with the same effect.

${ }^{6}$ An alternative approach, as shown by Schlag (1994), is to suppose that individuals come from two different populations. In that case evolutionary stability can lead to efficient outcomes even with a finite message space.
} 
is no limit to the number of correct expressions that may be constructed. Yet in communicating with another person we choose a finite message from this infinite set.

Note that gifts are costly to give but confer no benefit to the receiver. In Section 3 we will argue that gift goods should have low use value, but at this stage the assumption is just a normalization -i.e. the amount $g^{i}$ is the difference between the cost of the gift and its value to the receiver. Our analysis will show that $g^{i}$ must be positive in a cooperative equilibrium.

In assumption 6 we require that agents die in pairs only to simplify the computation of the equilibrium payoffs. Finally we suppose that the history of a match is private information to the two participating parties, and agents use strategies that are contingent only on the history of their current match. Given that there is a continuum of agents the probability of meeting the same agent again is zero, and hence once separation has occurred knowledge of this match is irrelevant.

Agent $i$ 's expected payoff in period $s$ is given by $V_{s}^{i}$. The present expected value of payoffs to agent $i$ entering the population in period zero is

$$
V^{i}=\sum_{s=0}^{\infty} V_{s}^{i} \rho^{s}
$$

In what follows we suppress the superscripts $i$ and $j$ wherever possible.

A strategy, denoted by $\sigma \in S$, is a collection of messages and actions (including separation decisions) used by an agent in his matches. Agents do not play mixed strategies, although there may be a mix of strategies present in a population. Let $E$ denote a population and let $S_{E}$ denote the set of strategies in use in population $E$. The population profile $\widetilde{E}$ is a vector with generic element $p^{\sigma}$, where $p^{\sigma}$ is the proportion of agents in the population playing strategy $\sigma$. The set of all possible population profiles is denoted by $\Delta(S)$.

The set $\Delta(S)$ is restricted in two important ways. First, as we have already assumed, the number of messages sent by any strategy is finite, although the set $M$ of possible messages is not. Second, we assume that for all $\widetilde{E}$ in $\Delta(S)$, positive weight is placed on at most a finite number of strategies. These two assumptions ensure that in any population there exist unused messages that allow new strategies to enter and signal their intentions. 
They also allow us to avoid the use of measure theoretic arguments. ${ }^{7}$

We wish to characterize stationary equilibria with time invariant individual strategies $^{8}$. Suppose at the beginning of the game all agents are in the market, with $\widetilde{E}$ describing the stationary distribution of strategies. Those agents who enter into relatively long term matches will re-enter the matching market at a slower rate than the others. It follows that the distribution of strategies that are available to form matches at the beginning of each period will be different from $\tilde{E}$. We assume that the distribution of strategies in the matching market converges to a stationary distribution, denoted by $m(\tilde{E})$, called the market profile. We do not provide a full characterization of the conditions for the existence and uniqueness of $m(\tilde{E})$, but we do provide some sufficient conditions for finite populations in an appendix.

The payoff to an agent playing strategy $\sigma$ who enters the population $\tilde{E}$ is determined by the distribution of agents available in the matching market. We denote this payoff by $V^{\sigma}(m(\tilde{E}))$. Finally, Let $E^{\sigma}$ denote population where all agents play the strategy $\sigma$.

\subsection{Nash Equilibria}

A necessary condition for a population to be an equilibrium is that it must form a Nash equilibrium.

Definition 1 The set of best replies to a population profile $\widetilde{E}$ is defined by:

$$
B R(\widetilde{E})=\left\{\sigma \in S \mid V^{\sigma}(m(\widetilde{E})) \geq V^{\sigma^{\prime}}(m(\widetilde{E})), \forall \sigma^{\prime} \in S\right\} .
$$

\footnotetext{
${ }^{7}$ We measure the distance between two populations with the following metric:

$$
d\left(E_{1}, E_{2}\right)=\sup _{\sigma \in S_{E_{1}} \cap S_{E_{2}}}\left|p_{1}^{\sigma}-p_{2}^{\sigma}\right|+\#\left\{\left(S_{E_{1}} \cap S_{E_{2}}\right)^{c} \cap\left(S_{E_{1}} \cup S_{E_{2}}\right)\right\}
$$

The second term simply counts up the number of strategies the two populations do not have in common. With this metric the convergence of one population to another requires that the support be the same and that there is convergence of the proportions of each strategy in the populations. Thus when we speak of convergence of populations we do not need to consider new strategies.

${ }^{8}$ A time invariant population distribution does not imply time invariant individual strategies. For example each period half of the population could switch strategies with the other half. There is no benefit to this type of behavior in the model, so it is excluded from discussion.
} 
A population profile $\widetilde{E}$ forms a Nash equilibrium iff $S_{E} \subseteq B R(\widetilde{E})$.

Our first result shows that a cooperative Nash equilibrium in the absence of gift exchange does not exist.

Proposition 2 Cooperative play by all agents in every period in the absence of gift exchange is never part of a Nash equilibrium strategy.

Proof. Suppose that the population profile $\widetilde{E}$ is a Nash equilibrium resulting in cooperation every period. The equilibrium payoff for all strategies in this profile must be:

$$
V(m(\widetilde{E}))=\sum_{t=1}^{\infty} A \cdot \rho^{t}=\frac{A}{1-\rho} .
$$

To achieve this outcome there may be a set of messages that are sent at the beginning of each match that results in the partner choosing the cooperative strategy at stage 4 . Consider now the "parasitical" strategy of sending this message, choosing to play B at stage 4 and quitting at stage 5 . Denote this strategy by $\sigma^{P}$. The payoff from following this strategy is given by:

$$
V^{\sigma^{P}}(m(\widetilde{E}))=\sum_{t=1}^{\infty} B \cdot \rho^{t}=\frac{B}{1-\rho}>\frac{A}{1-\rho} .
$$

Therefore the original cooperative market profile could not have been a Nash equilibrium.

This proposition restates the message from the introduction - even though the game is indefinitely repeated, the opportunity to quit and form new matches prevents full cooperation. The result is quite different from the more positive conclusions of Axelrod (1984) and Maskin (1993), both of whom use the round robin framework. The ability to communicate does not facilitate cooperation here since parasites can mimic any signal that induces cooperative behavior in the partner. It is also clear that since $\mathrm{D}$ is a dominant strategy, the population profile in which agents play D every period (and may or may not quit the relationship) is a Nash equilibrium. This is stated in the next proposition.

Proposition 3 There exists a Nash equilibrium in which no gifts are exchanged and all agents defect every period. 
There may also exist Nash equilibria in which some of the agents play cooperative strategies and some do not. Before we establish this, it is useful to define more formally two classes of strategies, denoted by "clubby" and "parasite". We will make use of these strategies throughout the paper. We begin with the description of a "clubby".

Recall that $M^{i}$ is the set of messages sent by agent $i$ to his partner $j$, and $m^{i}\left(\ell_{i}\right)$ is the maximal message sent by $i$. Agent $i$ can therefore recognize only the signals $m^{j}(\cdot) \in\left\{m(1) \ldots m\left(\ell_{i}\right)\right\}$. Let $M_{i}^{j}$ be the message sent by $j$ as perceived by $i$. A group of agents can form a club by coordinating on the choice of messages. Let $M^{c}$ be the agreed upon message. Club member $i$ will therefore play the strategy given by

$$
\sigma^{C, M}=\left\{\begin{array}{cc}
\text { stage } 2 & \text { send message } M^{c} \\
\text { play } C \text { if } M_{i}^{j}=M^{c} \\
\text { play } D \text { if not } \\
\text { stage } 7 & \text { stay if } M_{i}^{j}=M^{c} \text { and } C \text { is chosen } \\
& \text { separate if } M_{i}^{j} \neq M^{c} \text { or if } D \text { chosen by the other player }
\end{array}\right.
$$

This strategy incorporates the idea that players cooperate only with other agents that send the appropriate messages. Of course there may be an incentive for another agent to enter, send the same signal, choose D and then quit. We have called such an individual a "parasite", with a strategy defined by:

$$
\sigma^{P, M}=\left\{\begin{array}{cc}
\text { stage } 2 & \text { send message } M^{c} \\
\text { stage } 4 & \text { play } D \\
\text { stage } 7 & \text { quit }
\end{array} .\right.
$$

Equilibria with a mix of parasites and clubbies can exist because a clubby, when it meets another clubby, leaves the matching market until it dies. Parasites remain in the market forever. The proportion of parasites in the market is therefore higher than the proportion in the population, and this reduces the return to being a parasite.

Proposition 4 Suppose that the payoff parameters satisfy the following condition:

$$
((A-B)+(1-\rho B))^{2}-(4 \rho(B-A)(B-1)) \geq 0 .
$$

Then there exists a Nash equilibrium with no gift exchange where a strictly positive fraction of the population plays cooperatively every period. 


\section{Proof. See the appendix.}

In general, when partially cooperative equilibria exist there will be two of them, with the one having the smaller proportion of parasites being locally stable and the other unstable.

\subsection{Evolutionarily Stable Equilibria}

Evolutionary stability, although its origins are in biology (Maynard Smith (1982)), can be interpreted as a refinement of Nash equilibrium. It requires, in addition to the Nash properties, that a small but significant group of agents with a new strategy will do worse than the incumbents if it tries to enter a population.

Evolutionary stability is a sufficient condition for an outcome to be asymptotically stable under a wide variety of selection dynamics. A selection dynamic is a process that determines next period's population profile as a function of the current one. Though we do not explicitly model the dynamics of our model, we can give a brief description of a process which might underlie it. Each period when the new generation of individuals enter, most simply mimic the strategies of their parents. A smaller fraction change their strategies to mimic the existing strategy with the highest expected return. An even smaller fraction experiments with randomly chosen strategies. In this way the entry of mutations each period constantly pushes the population off the equilibrium. Evolutionary stability guarantees that the players who make optimal choices push the population back to the equilibrium. Excellent references on the relationship between evolutionary stability and selection dynamics include Taylor and Jonker (1978), Hofbauer and Sigmund (1988) and Van Damme (1991).

In what follows we will be comparing the payoffs to strategies from an incumbent population $E$ and a small invading population $E^{\prime}$. For $\epsilon \in[0,1]$ the combination of these two populations will have the profile $(1-\epsilon) \widetilde{E}+\epsilon \widetilde{E}^{\prime}$, with generic element $(1-\epsilon) p^{\sigma}+\epsilon p^{\sigma}$ and where $p^{\sigma} \equiv 0$ for $\sigma \notin S_{E}$ and $p^{\sigma^{\prime}} \equiv 0$ for $\sigma^{\prime} \notin S_{E^{\prime}}$. Suppose that the proportion of the agents in $E$ that are in the matching market each period is given by $\delta \in[\rho, 1]$. The period 
that $E^{\prime}$ enters, the strategy profile in the matching market will be

$$
\frac{\delta(1-\epsilon) m(\widetilde{E})}{\delta(1-\epsilon)+\epsilon}+\frac{\epsilon \widetilde{E}^{\prime}}{\delta(1-\epsilon)+\epsilon}
$$

Sometime afterwards, it will converge to $m\left((1-\epsilon) \widetilde{E}+\epsilon \widetilde{E}^{\prime}\right)$. We assume that the rate at which this convergence occurs is much faster than the rate at which the selection dynamic operates. In other words, it is appropriate to compare the performance of strategies from $E$ and $E^{\prime}$ in the stable market profile for the mixed population. ${ }^{9}$

Evolutionary stability normally requires that agents using any strategy that is not part of the equilibrium be strictly worse off than incumbent agents. But in our model the space of possible messages is unlimited and signaling is costless. In any given population it is always possible for an agent to duplicate an existing strategy and also send a message that is not observed by any other member of the population. Formally this is a new strategy, not a part of the equilibrium, and clearly it will result in the same payoff as the strategy it is copying. Evolutionary stability, therefore, can never be satisfied when the space of communication possibilities is unlimited. ${ }^{10}$

We resolve this problem by using the concept of neutral stability due to Maynard Smith. Formally, we have:

Definition 5 A population profile $\widetilde{E} \in \Delta(S)$ forms a neutrally stable equilibrium (NSE) if:

1. (Nash Equilibrium) $S_{E} \subseteq B R(\widetilde{E})$

2. (Stability) For $\widetilde{E}^{\prime} \in \Delta(S) \backslash \widetilde{E}$, there exists an $\bar{\epsilon}>0$ such that for all $\epsilon \in[0, \bar{\epsilon})$ :

$$
V^{\sigma}\left(m\left((1-\epsilon) \widetilde{E}+\epsilon \widetilde{E}^{\prime}\right)\right) \geq V^{\sigma^{\prime}}\left(m\left((1-\epsilon) \widetilde{E}+\epsilon \widetilde{E}^{\prime}\right)\right), \forall \sigma \in S_{\tilde{E}}, \sigma^{\prime} \in S_{\tilde{E}^{\prime}}
$$

Condition 1 is the requirement that the population form a Nash equilibrium. The second is the stability condition. If a small "mutant" population, given by $E^{\prime}$, enters then all members of this population will be no better off in the new mixed population than the

\footnotetext{
${ }^{9}$ The stability of the market profile is discussed in the appendix.

${ }^{10}$ The problem is common to nontrivial extensive form games. See Kim and Sobel (1992) for an excellent discussion of these issues
} 
agents using strategies from the original population. The definition is constructed to include the possibility that a single mixture be stable, i.e. if the proportion of a given strategy increases, then this strategy must be made no better off relative to the other strategies. It differs from the stronger notion of an evolutionarily stable equilibrium where the inequality is required to be strict.

The next two results underline the effects of free communication.

Lemma 6 If free communication is not permitted then the Nash equilibrium of defect every period is neutrally stable regardless of the matching strategy.

Proof. The result is immediate since any strategy using either costly communication (gifts) or some level of cooperation results in a payoff against a defect strategy that is strictly less than the defect strategies payoff. This implies that one can always make $\bar{\epsilon}$ small enough in the definition of an NSE to ensure stability.

Lemma 7 With free communication, at any neutrally stable population all agents choose cooperate in stage 4 .

Proof. Let $\widetilde{E}$ be neutrally stable. Suppose that in this profile there are strategies that result in the choice of $D$ with positive probability. Now consider the following set of entrants called cooperators. For each $\sigma \in S_{E}$, let the corresponding cooperator be $\sigma^{c}$. A cooperator follows exactly the same matching rules as the strategy from which it is derived. However, whenever it is matched with another cooperator, it plays $C$. To do this the cooperators use messages that are not observed by the equilibrium population.

A cooperator $\sigma^{c}$ uses the same matching strategy as $\sigma$ even if matched with another cooperator. If $\sigma$ separates after one of the players chooses $D$, we suppose that when $\sigma^{c}$ meets another cooperator it sends a signal representing $D$ when the original strategy requires this, but still chooses $C$. The other cooperator uses the message representing $D$ to duplicate the matching behavior required by the strategy upon which it is modeled. This ensures that the introduction of these cooperators does not affect the distribution of 
matches in the population (with the cooperators identified with the strategies from which they are derived).

Now consider the population profile $\widetilde{E}^{c}$ of cooperators with the proportion of each $\sigma^{c}$ equal to the proportion of $\sigma$ in $\widetilde{E}$. If $E^{c}$ invades $E$, our construction ensures that $\sigma$ and $\sigma^{c}$ together will form the same proportion of the new population profile and the new market profile as $\operatorname{did} \sigma$ alone in the equilibrium profiles. It follows that for all $\epsilon \in[0,1]$ we have the following equality:

$$
V^{\sigma}\left(m\left((1-\epsilon) \widetilde{E}+\epsilon \widetilde{E}^{c}\right)\right)=V^{\sigma}(m(\widetilde{E})), \forall \sigma \in S_{E}
$$

It has been assumed that $2 A>B$, so given that $\mathrm{D}$ is chosen with positive probability, and since all strategies must get the same payoff in any equilibrium, the payoff to any incumbent strategy must satisfy:

$$
V^{\sigma}(m(\tilde{E}))<\frac{A}{1-\rho}-c\left(g^{\sigma}\right)
$$

where $c\left(g^{\sigma}\right)$ is the cost of any gift giving actions by strategy $\sigma$. When a cooperator is facing another cooperator it receives $A$ per period, therefore given an $\epsilon>0$, there is a $\gamma \in(0,1)$ such that:

$$
V^{\sigma^{c}}\left(m\left((1-\epsilon) \widetilde{E}+\epsilon \widetilde{E}^{c}\right)\right)=\gamma\left(\frac{A}{1-\rho}-c\left(g^{\sigma}\right)\right)+(1-\gamma) V^{\sigma}(m(\widetilde{E}))>V^{\sigma}(m(\widetilde{E}))
$$

In particular the strategy profile $\tilde{E}$ is not an NSE.

Since we know from Proposition 2 that cooperation is never a Nash equilibrium in the absence of gift exchange, an immediate consequence of this lemma is the non-existence of a neutrally stable population.

Proposition 8 In the absence of gift exchange there are no neutrally stable populations.

Nonetheless, we will now describe a method of sustaining cooperation that seems to have been known to human societies for many generations. Suppose that at the beginning of the relationship agents send messages as before, but now may also incur the cost $g^{i}$ to send a gift. At this stage the only properties a gift must have is that it be visible and costly. 
Proposition 9 For $(B-A) /(B-1)<\rho<1$ there exist neutrally stable populations, all with strategies that have the following characteristics:

1. Individuals exchange gifts once at the beginning of the match, stay matched until death, and play cooperate every period.

2. All agents send the same sized gift that is bounded as follows:

$$
\frac{A-1}{(1-\rho)}>g>\frac{B-A}{\rho} .
$$

Proof. We argue first that any population $\widetilde{E}$ that is an NSE will satisfy properties 1 and 2. From Lemma 7 we know that agents cooperate every period. Furthermore, to stop entry by parasites, agents must give a gift at the beginning of each relationship. The matching is anonymous, therefore this gift is made unconditionally. Let $\sigma \in S_{E}$ be a particular strategy that involves some turnover for reasons other than death, or further gift giving within its matches. It must be the case that $V^{\sigma}(m(\widetilde{E}))<A /(1-\rho)-g^{\sigma}$, where $g^{\sigma}$ is the gift given under strategy $\sigma$ at the beginning of a new match.

As in the proof of lemma 7 define a cooperator strategy $\sigma^{c}$ that is identical to $\sigma$ except that when it meets itself (identified by sending secret signals) it cooperates, stays matched until death, and gives no further gifts. When it meets itself it will get the payoff $A /(1-\rho)-g_{\sigma}$. Note that if $\sigma$ calls for any extra turnover, $\sigma^{c}$ will be less likely to enter the matching market than $\sigma$.

Now consider an invading population identical to $E$ except that all the agents playing $\sigma$ are now playing $\sigma^{c}$. In the new mixed market profile the total fraction of $\sigma$ and $\sigma^{c}$ types in the market each period will be no greater, and perhaps less, than the fraction of $\sigma$ types in the original market profile. This is because the $\sigma^{c}$ stay matched forever when they meet their own type, while the $\sigma$ strategies may experience early separation. ${ }^{11}$ Now increase the proportion of $\sigma^{c}$ types until the fraction of $\sigma$ and $\sigma^{c}$ types in the matching market is equal to the fraction of $\sigma$ before invasion.

\footnotetext{
${ }^{11}$ Since all strategies stay matched at least one period, the outflow from the matching market does not vary across strategies. Those that are more likely to enter thus make up a larger proportion of the matching market.
} 
This construction ensures that the introduction of cooperators does not change the payoffs to any of the original strategies in $E$. However cooperators have a positive probability of meeting each other and thus earn a payoff that is strictly greater than the payoffs to the other strategies. Consequently the original population could not have been an NSE, and neutral evolutionary stability implies gift giving at the beginning and a match forever.

It is now immediate that in equilibrium all strategies must send the same sized gift, else they would not be getting the same payoff.

For the rest of property 2, we note first that the market profile must form a Nash equilibrium. Any parasites that enter, give the gift (to hide their true intentions) and cheat must fare no better than the incumbents. This implies:

$$
\frac{A}{(1-\rho)}-g \geq \frac{B-g}{(1-\rho)},
$$

which immediately gives $g \geq(B-A) / \rho$. Suppose this condition holds with equality. Then a secret society of parasites could enter, give gifts, and then cooperate with each other but defect against everyone else. Members of this group would get $A /(1-\rho)-g$, but incumbents would get less since there is a positive probability that they would meet a parasite. Stability therefore requires that the inequality be strict.

Similarly, to ensure that agents are not better off giving no gifts at all, we must have

$$
\frac{A}{(1-\rho)}-g \geq \frac{1}{(1-\rho)}
$$

which immediately gives $(A-1) /(1-\rho) \geq g$. As before, suppose this condition holds with equality. Then a group of agents could enter who give no gifts but cooperate among themselves. They will do better than the incumbents who give gifts, and it follows again that the inequality must be strict.

Finally we need to exhibit a collection of strategies which form a neutral evolutionarily stable population. Consider the following class of clubby strategies, where all agents use 
the same size gift, and $g$ satisfies condition 11 with strict inequality:

$$
\sigma^{C, g}=\left\{\begin{array}{cc}
\text { stage } 2 & \text { give gift } g \text { and send any finite set of messages } \\
\text { stage } 4 & \text { play } \mathrm{C} \text { if gift equal to or larger than } g \text { is given } \\
\text { stage } 5 & \text { stay } \mathrm{D} \text { if not } \\
& \text { quit if not }
\end{array}\right.
$$

Let $\widetilde{E}$ be a population profile where all agents play this strategy. Note that the agents need not coordinate on a set of costless messages, although they all give the same sized gift. Due to the strict inequality in 11, any strategy outside this class results in a payoff that is lower or equal when played against the population of clubbies. The only way that an invading population could do strictly better is by agreeing to send a smaller gift. But this will always result in punishment from a clubby and a payoff that is strictly less than the clubby payoff. Consequently such an invasion is not profitable.

An important assumption used in the proof is that gifts and messages must come at the same time. If (secret) messages can come before gifts, then a group of agents could enter who always cooperated but agreed not to exchange gifts among themselves. This group would do well enough initially to break the equilibrium, but would eventually be invaded by parasites. We discuss this case informally at the end of Section 3

The message sending technology is important in the proof, although it does not seem to matter in the equilibrium. All that matters is that agents give gifts. Of course there are other equilibria where the entire population also coordinates on a set of costless messages. Communication is important, however, in ruling out some equilibria. If agents could exchange gifts but nothing else before a match, then by Lemma 6 the all defect outcome would also be an $\mathrm{NSE}^{12}$.

\footnotetext{
${ }^{12} \mathrm{It}$ is also worth emphasizing why this equilibrium is not evolutionarily stable in the usual sense. Evolutionary stability requires that invading strategies get strictly lower payoffs. However, in equilibrium a strategy that (for example) duplicates the clubby strategy but does not bother to quit when it is defected upon earns the same payoff as a pure clubby strategy. We thank Akihiko Matsui for pointing this out.
} 


\subsection{Some Principles of Gift Giving}

The preceding analysis has shown that a social convention requiring the use of gifts at the beginning of a relationship can support long term cooperation. The next section goes on to discuss the character of gifts in more detail. At this point it is useful to summarize the results so far. Assume for now that the costly messages above really are gifts. Then:

1. Gifts that are exchanged between the two parties should be inefficient. If the partners exchanged money at the beginning of a match, then parasites could immediately enter, give (and receive) the money, cheat, and move on.

2. When matches are very long term (i.e. when $\rho$ is close to 1 ), the cost of the gift can be as little as $B-A$, the one-time benefits of cheating. This may be far less than $(A-1) /(1-\rho)$, the overall benefits of cooperation. Gifts will be more expensive when the life of a match is short. If matches are too short (i.e. $\rho$ gets close to zero), then the conditions of Proposition 9 cannot be satisfied, and gift giving will not help to achieve cooperation. Gift giving customs will surround only the important (i.e. A-1 large), long term relationships in people's lives.

3. Gift giving is a fully decentralized institution - i.e. the fact that a gift was given need be verified only by the other partner to the match. In this way it may be a low cost substitute to reputation mechanisms that require more public information on the behavior of agents in their previous relationships.

4. Gifts are a universal language. Members of a gift giving society can indulge in all of the costless message sending they wish, so long as they also give gifts. This result contrasts with those found in similar matching models where repeated interaction with one partner is not possible (e.g. Gauthier, 1986, Frank 1988). In those models there are equilibria where agents cooperate only with others they perceive to be just like them. Here we get the more optimistic conclusion that when meeting strangers who seem very different, up front gifts can be the passage to cooperation and understanding. 


\section{Gifts}

Several question remain. First, why should costly messages take the form of gifts? The amount $g$ in Proposition 9 could just as well be extracted via physical punishments. Second, what predictions, if any, does the model make about the form of the gifts that should be exchanged? And are these predictions precise enough to distinguish the model from other explanations for gift giving?

\subsection{Efficiency and Empirical Implications}

Exchange gifts are a socially efficient way of sending a privately costly message. This is best understood with reference to Figure 1.

Figure 1 here.

Suppose a society has agreed that good $G$ is to be used as a gift good. This good has a constant use value of $v$ and in competitive equilibrium a price equal to $v$ and quantity $G_{c}$. Gift status raises the demand for the good so that price is $P$ and quantity $G_{g}$. The cost of the message sent by use of this gift good is the excess of price over use value (since the gift is received as well as sent) times the quantity purchased. In aggregate, the total excess payments $G_{g}(P-v)$ are equal to the flow of new matches times $g$. However, the social cost of the arrangement is far less - it is just the usual triangle between the supply curve for good $G$ and the horizontal line at $v$ between $G_{c}$ and $G_{g}$. The rest is a transfer to the gift makers. If the supply curve is vertical then the social costs of gift exchange are exactly zero. ${ }^{13}$

While a gift giving population might achieve full efficiency, it does not necessarily follow that a small group playing this strategy could successfully invade a population that achieved cooperation by using some other socially wasteful technique. The invading gift givers would not get cooperation if matched with incumbents, and thus the invasion would

\footnotetext{
${ }^{13}$ With the usual caveats about partial equilibrium.
} 
fail. ${ }^{14}$ Allowing secret messages to come before gifts would allow the invasion to succeed, but introduces other complications that will be explored at the end of this section

Indeterminacy like this is endemic to evolutionary models. It is not so much a problem to be resolved as much as a reflection of the incredible diversity of global customs (and ecosystems). Further progress can be made, we suspect, but will require a much better understanding of the actual process by which new customs enter a population. ${ }^{15}$

However our model is still able to make a great many predictions. Our major hypothesis is that the gift giving conventions we observe are social customs that have arisen to facilitate cooperation, and have survived years of evolutionary competition. To have done so, they must have been able to fend off challenges from other invading strategies.

There are two strategies that seem most likely to invade a population of gift givers. The first would be followed by a "parasite consumer", who routinely consumes the gift good. Such an agent, in anticipation of a match, could cut back on her normal purchases of the gift good. Since gifts are exchanged, the marginal cost of gift giving to this agent is zero, and the parasite "give gift, defect and quit" strategy is optimal. The second invader might be called a "parasite recycler". This agent simply takes the gift received from a match, defects, and then uses the received gift to start up a new match. Again the marginal cost of gift giving (after the first purchase) is zero, so this defect and quit strategy can invade.

If a good has achieved gift status, and if our model is right, then the good should have properties that make invasion by these strategies difficult. This observation allows us to predict a great deal about gift goods. First, in order to repel "parasite consumers", it is important that the normal demand curve for the good lie below its gift price even at very low levels of consumption, as in Figure 1. Thus the gift good cannot be an essential commodity, and indeed an entirely useless commodity might make a fine gift good.

\footnotetext{
${ }^{14}$ Similarly, in Proposition 2.9 evolutionary stability does not imply that the cost of the gift will be in the lower end of its range. An invading group with a lower cost gift, even if this gift satisfied the conditions of the proposition, would be rejected by the incumbent population.

${ }^{15}$ If there are agents in the population who have a lot to gain from the establishment of a custom, and if the custom is evolutionarily stable, then a small mount of persuasion could generate big returns. Perhaps the profit opportunities available to gift makers have had some impact.
} 
Since it is important that the receiver be able to verify the cost of the gift, the normal practice of removing the price tag seems to be a puzzle. But another way to consume a gift good is to take it back to the store for a refund. Removing the price tag from a gift helps to prevent this. Since the price of a conventional gift good is sure to be known anyway (or readily obtainable) it is hard to believe that price tags are removed to hide information. Note that when people give wedding gifts to a young couple (a convention not addressed by this model), often price tags and even receipts are included. Money is also sometimes given directly.

The prevention of recycling can be accomplished in several ways. One is to choose as the gift good a commodity which depreciates rapidly. Cut flowers is the obvious example, but there are others. Gifts are often wrapped, and indeed in Japan much of the expense of a gift is in the packaging and presentation. A gift which is mostly a nice (personalized) package depreciates completely the moment it is opened. A consumable with a longer shelf life, such as a bottle of wine, can be used if the custom specifies that it be consumed in the giver's presence.

Another option is to designate the direction of the giving. If matches are always between members of the opposite sex, for example, then custom can specify which gifts are given by each partner. Diamonds might be the most durable good there is, but they make excellent gift goods so long as it is the man who gives and the woman who receives. ${ }^{16}$ Even when rings are exchanged the differences in style and average size will prevent recycling.

Durability can still cause problems, however, if there is an active resale market. If the woman could sell the diamond for its appraised value, or if the man could buy one easily on the resale market, then again parasitical strategies could invade. In fact, the market for used diamond rings is extremely thin, as a perusal of the classified advertisements in any newspaper will attest. (Given current divorce rates, there is no shortage of supply.) The market is actively discouraged by retailers, who will gladly appraise rings for high value, but will not buy them for anything near that price, if at all. ${ }^{17}$ Also, the custom itself

\footnotetext{
${ }^{16}$ Abigail Van Buren ("Dear Abby"), my authority on these matters, has stated that diamonds are an unconditional gift. Even if the woman later breaks off the engagement, she is entitled to keep the ring. But it is not of much use to her, and Abby does suggest it would be nice to give it back.

${ }^{17}$ Dan Usher has told us that the original "Diamonds are Forever" advertising campaign in the 1930's
} 
discourages buying diamonds on the cheap.

The model in Section 2 is completely symmetric. In a pure "double standard" world, where only one party has anything to gain from cheating, the model predicts that gifts need only be given by that party. Recycling will not be a problem, but the receiver is still in a position to quit in order to collect more gifts. Thus the gifts should have low use value, be difficult to sell, and the rest as above.

While our model predicts that gift giving customs should surround important and long term relationships, this does not limit it to the marriage market. In the business world, despite the obvious reliance on explicit contracts to ensure performance, the beginning of new relationships is often marked by gifts of various kinds. Overpriced dinners, and the donation of time from people whose time is expensive are examples. Gifts and explicit contracts seem to be substitutes. In Japan, where lawyers are less influential, a great deal of time must be invested by both parties before talk of business even begins.

Our model addresses gift giving at the beginning of relationships. Gifts are often exchanged in mature relationships as well. Most of this is probably outside the model, although there is a situation in which the model may apply. It is now well known that possibilities for renegotiation can prevent the attainment of cooperative equilibria in repeated games. The idea is formally very similar to the matching story of this paper. A player can defect, and then avoid punishment by offering to renegotiate. A social convention that any renegotiation must begin with some inefficient gifts from the transgressor can prevent this problem. Some gift giving in mature relationships may be part of an attempt to "start the relationship anew" and can therefore be explained within our model.

The beginning of a new match is often marked with celebrations and feasts. This is also a gift giving custom, but here the gifts are given to third parties like friends and family members. Since these agents are not parties to the match, it seems as if these gifts could be efficient. However, as with exchange gifts, one must consider new strategies that might invade a society which gives third party gifts. Again, this is the source of some empirical implications.

suggested, quite literally, that once you buy a diamond, you should keep it forever. 
Suppose there was a population where each agent in a new match marked the occasion with a donation of money to one or more acquaintances. First, it is clear that this donation must be observable to the other partner. Otherwise a group could enter whose members only pretended to give gifts to each other. These agents could defect in their relationships with outsiders and break the equilibrium. Similarly, a group could enter making public gifts, but secretly give them back to the donors afterwards. Members of this group could also profitably defect, breaking the equilibrium as before. One way to prevent this is to make the gifts consumable, and to have their consumption be a public event as well. But the "public donation of consumables to one's friends" is of course just a rather formal way of defining a big party.

A key assumption in our model is that gifts must come right at the beginning, at the same time as any other messages. When secret messages can come before gifts, there is no equilibrium in the usual sense. Any gift giving society can be invaded by another using less expensive gifts. There is no least expensive gift, given the strict inequality in condition (11). But as these gifts get smaller, the new society can be invaded by parasites. A society of parasites can be invaded by a group giving expensive gifts.

Although there is no equilibrium population profile the model still makes some predictions about gift giving. Suppose that smaller groups enter more readily, and parasites the most readily of all. Consider a strategy that uses gifts close in size to the lower bound in Proposition 9. This society will eventually be invaded by a secret society giving less expensive gifts. The cheaper the gift, the more rapidly the invader will begin to grow, relative to the incumbent. But the cheaper the gift the more quickly the invader will itself be invaded by parasites. If the parasites enter quickly enough, the original strategy may survive for a long time, and gift giving customs will be observed.

More important, in order to repel parasite consumers and recyclers, any surviving strategy must have choosen gift goods that have low use value, depreciate rapidly, and all the rest as outlined above. Pre-gift communication, while it destroys the existence of equilibrium, leaves many of the model's implications intact. 


\subsection{Other Explanations}

Several other explanations for gift giving have appeared in the economics and biological literatures. ${ }^{18}$ The first is pure altruism (Becker 1981), which suggests that gifts are chosen to maximize the giver's altruistic utility within a fixed budget. Christmas giving and giving within families is probably well described by this model, as is the giving of gifts to young couples on the occasion of their marriage.

An alternative is the reciprocity model introduced to economics by Akerlof (1983), and examined in great detail by Mauss (1990). Gift giving here is seen as a natural extension of the process of exchange. When one hands over money to buy something at a store, she seldom worries that the store owner will refuse to hand over the purchased articles. The obligation to reciprocate is so strong we take it for granted. In many societies, such obligations can stretch over many months or years, and become a store of wealth and a source of insurance. This approach also suggests that gifts be purchased at minimum cost conditional on satisfying the constraint that the obligation be fulfilled. Neither of these first two approaches, therefore, suggests that gifts should be inefficient. Neither do they suggest that conventional gift goods should be used, or that gifts be given to third parties.

More recently, Camerer (1988) has argued that gifts can be a signal of good intentions. ${ }^{19}$ Only those people who foresee genuine gains from a long term relationship will be willing to start it with an expensive gift. This is a rich and interesting analysis of gift giving which makes some of the same predictions as ours. The structure of the model is quite different, however, as are some of the implications.

In Camerer's model there are two types of people who may enter a one period, simultaneous move match. For an "unwilling" type, cheating is a dominant strategy. A

\footnotetext{
${ }^{18}$ Dawkins (1976) builds a model where some females insist on a long period of courtship to screen for "domesticity" in their mates. His model is cast in an evolutionary framework, but the strategy space is severely restricted. Males can play only "court/stay" or "don't court/quit". "Court/quit", which is analagous to our "parasite" strategy would invade his equilibrium. There is also the old general equilibrium example where an agent can be made better off by giving up some of his endowment.

${ }^{19}$ In a similar manner, forward induction arguments (Van Damme (1989) suggest it can be optimal for an agent to burn money at certain stages in a game. Formally, forward induction is a technique for selecting among equilibria. Gift giving in our context allows for the existence of a cooperative equilibrium.
} 
"willing" type will want to cooperate if and only if her partner is also willing. Each partner knows the proportion of willing types in the population, but does not know her partner's type. If the proportion of willing types is high enough, a willing type may cooperate in the simple hope that her partner is also willing. If this proportion falls, a willing type may in a previous stage give a gift to signal her type. This gift will cost no more than the expected benefits from cooperating, but must cost more than the expected benefits to an unwilling type of cheating on a cooperative partner. Since only a willing type will want to pay for a gift this size, the gift is an accurate signal of type.

The inefficiency of gifts is not a central part of this model. Since the signaling game is simultaneous move Nash, in equilibrium each player expects to receive a gift with some probability regardless of his own action, and the choice of any personally costly gift will accurately signal type. To get inefficiency, Camerer introduces a preliminary stage where players must pay some small cost to enter the signaling game. Then, to prevent people from cynically paying this cost, receiving a gift (but not giving one), and then running away, the use value of the gift must be small. ${ }^{20}$ To emphasize, however, the signaling model by itself does not predict inefficient gifts, and does not suggest anything about the character of the goods that societies will choose to use as gifts. ${ }^{21}$

The implications of the models differ in several other ways. In the signalling model, gift giving is not a widespread phenomenon. Only the willing types give gifts, and if the proportion of willing types in the population is sufficiently high, then nobody gives gifts. Second, Camerer claims the signalling approach cannot explain why price tags are customarily removed from gifts. In the repeated environment of our model it becomes natural to think of recyclers and consumers, and the removal of price tags makes evolutionary sense.

Finally, in our model gift giving is truly a social custom. People wrap gifts, remove price tags, and throw expensive parties without really knowing or caring why. It is just the

\footnotetext{
20 "Parasites" in Camerer's model and ours behave differently. In ours, they enter a match and then cheat. In his they take the gift and run.

${ }^{21}$ Camerer is forced to rely on undefined "social convention" to explain why societies tend to focus on particular goods to use as gifts. He suggests that these goods depreciate rapidly since this makes it more difficult for the recipient to consume them at the optimal time, adding to their inefficiency.
} 
right thing to do given the occasion. Agents in our model are capable of interesting, sophisticated, and complicated behavior even though they are not perfectly rational in the usual economic sense. This aspect of the model follows directly from its evolutionary character, and we find it appealing.

Of course there are many different kinds of gifts and there may be equally many motivations for giving them. Our model, Camerer's, and the others are potentially complementary. We do not explore this here, but we do refer the reader to Camerer's paper for an engaging discussion of gifts and gift giving.

\section{Extensions and Conclusions}

We will conclude with some brief remarks about the evolutionary approach to the modelling of human behavior. Clearly the characterization of agents as completely predestined in their strategy choice is strange to most economists. We are more familiar with agents who are free to choose their strategies at each point in time

But clearly we are all influenced by the programming we receive from our parents and our culture, although we develop the capacity to override much of it as we mature. The approach we have taken here is an extreme case, in the same way the standard approach is an extreme case. But surely ours is the right extreme for the study of social customs such as gift giving. Social customs exist. As economists, all that we have lacked up to now is a model which recognizes this fact and yet has some empirical content.

If introspection is not enough, there is theoretical justification for the approach as well. Boyd and Richerson (1985) begin their discussion of the evolution of culture with the following argument. Suppose that human beings are a product of biological evolution. Suppose it is possible for biological evolution to create a gene which makes it possible for parents to imprint their children with rules of behavior which incorporate the adults' knowledge of the environment. Under what conditions would such a gene increase the chances for survival of the human species?

The requirements, briefly, are an environment which is sufficiently complex that sophisticated patterns of behavior are necessary and that the environment be changing 
over time (so that full genetic programming is not optimal), but not so quickly that the knowledge of the parents is of no value (or negative value) to the children. The reader is referred to Boyd and Richerson for the details, but these conditions do not seem particularly restrictive or unrealistic. The argument obviously applies to any species, and is therefore empirically quite testable.

The assumption that individuals are subject to cultural programming is on firm theoretical ground. The assumption that culture and custom are the outcome of an evolutionary process has genuine empirical content. Given these two facts, there is no longer any reason for economic discussions of "cultural norms" or "social conventions" to be admissions of defeat.

\section{Carmichael: Queen's University at Kingston}

MacLeod: Boston College and C.R.D.E., Université de Montreal

\section{Appendix}

\subsection{Existence and Stability of $m(\tilde{E})$}

In the text we have assumed that given an initial population profile $\tilde{E}$ the distribution of strategies in the matching market quickly reaches an equilibrium. There do exist non-stationary strategies (for example any aperiodic separation strategy) for which one clearly does not have convergence. In this section we demonstrate the existence and

convergence to $m(\tilde{E})$ for a finite population that approximates the equilibrium strategies studied in the paper.

Consider a finite population of individuals, indexed by $i \in N=\{1, \ldots, 2 n\}$. In our model at the beginning of each period agents are matched in pairs. Some of these matches last only one period, others are of longer duration. Given this pairing and the strategies associated to each player, one can deduce the probability that they will be matched with the same partner next period, and, if they separate, the probability that they will be paired 
with a new agent taken from $N$. The state space is the set of all pairings that is denoted by:

$$
\Pi=\left\{\begin{array}{c}
f: N \rightarrow N \mid f \text { is } 1-1 \text { and onto, such that } \\
f \circ f=\text { identity }, f(i) \neq i, i \in N .
\end{array}\right\}
$$

Observe that the size of the state space is $|\Pi|=(2 n-1) \cdot(2 n-3) \cdot \ldots \cdot 3 \cdot 1$. Consider the equilibrium case in which agents either separate after one period, or stay together until death (which occurs at a rate $\rho$ ). Let $\delta(i, j)$ be 0 if immediate separation occurs for a pair, and let it be 1 if they will stay together. In that case the probability of separation is $(1-\rho)$. All those agents that separate at the end of a period, are matched randomly at the beginning of the next period, resulting in a new state $s^{t} \in \Pi$ in period $t$. This implies that given any state $s^{t-1}=s_{k} \in \Pi$ (where $k$ is the index for a state taken from $\{1, \ldots,|\Pi|\}$ ) there is a well defined and fixed probability that state $s^{t}=s_{l} \in \prod$ will occur.

Let $P=\left[P_{k l}\right]$ be the transition matrix, where $P_{k l}$ is the probability of going from state $s_{k}$ to state $s_{l}$. Observe that given that there is a positive probability of death for each pair, then there is a strictly positive probability that all agents are in the market at the same time, and hence a strictly positive probability of occurrence for every state in $\Pi$. Thus $P$ is a strictly positive matrix, and by the Frobenius-Perron theorem there is a unique stationary distribution, $m$, with the property that $\bar{m} P^{t} \rightarrow m$ as $t \rightarrow \infty$. The speed of convergence is higher as $\rho \rightarrow 0$.

This limiting distribution can now be related to $\tilde{E}$ and $m(\tilde{E})$ in the text. Let $\pi(\sigma) \subset \Pi$ denote the set of individuals playing strategy $\sigma$. Then fraction of the population playing $\sigma$ is $p^{\sigma}=|\pi(\sigma)| / 2 n$. Given any profile $\tilde{E}$, because it consists of a finite number of strategies it can be approximated arbitrarily closely with a large $n$. The stationary distribution describes the distribution of the matches just after the anonymous matching in the market. Then the unconditional probability that individual $i$ is in the market at a stationary match distribution is given by:

$$
p^{i}=\sum_{f \in \Pi} m_{f}((1-\delta(i, f(i)))+(1-\rho) \delta(i, f(i)))
$$

where $m_{f}$ is the probability of state $f$ at the stationary distribution. The first term is brackets is 1 if agent $i$ is matched with a partner with whom separation is certain, while the second term is the probability of separation from a long term partner due to death. Let 
$q^{\sigma}$ denote the probability of strategy $\sigma$ being observed in the market for $m(\tilde{E})$, then it is defined as a function of $p^{i}$ and $\pi(\sigma)$ by:

$$
q^{\sigma}=\sum_{i \in \pi(\sigma)} p^{i} / 2 n .
$$

This illustrates that with a strictly positive death rate, the finite approximation to our model is assured of convergence to an equilibrium distribution of strategies in the market. This argument can be extended to include more general strategies, athough the details are somewhat more complicated. A sufficient condition is for matches of age $T$ and larger, where $T$ is some finite number, to have a constant probability of separation. In this case one increases the size of the state space by including the age of the match, with all matches of age $T$ and larger identified to be of the same age. This ensures that one has a finite state space with a time stationary transition matrix. This matrix will no longer be strictly positive because the transition from say a match of age 1 to age 5 would be impossible. However, due to the death process, there will be no absorbing states, with all states being either transitory or belonging to a single closed communicating class. Thus again there will be a unique equilibrium distribution $m(\tilde{E})$.

\subsection{Proof of Proposition 2.4}

Proof. We shall show that for any arbitrary message $M$, there exists a Nash equilibrium profile consisting of a mixture of clubbies and parasites. Let the fractions of parasites in the market at the beginning of each period be $\alpha$, and thus there is a fraction of $(1-\alpha)$ clubbies in the pool of unmatched agents at the beginning of each period. If one club member meets another then they will stay matched indefinitely and receive the payoff $A /(1-\rho)$. We have

$$
V^{C}=(1-\alpha) \frac{A}{1-\rho}+\alpha\left(0+\rho V^{C}\right)
$$

and

$$
V^{P}=(1-\alpha) B+\alpha+\rho V^{P}
$$

so that

$$
V^{c}=\frac{(1-\alpha) A}{(1-\alpha \rho)(1-\rho)}
$$


and

$$
V^{P}=\frac{(1-\alpha) B+\alpha}{(1-\rho)}
$$

In equilibrium $V^{C}=V^{P}$, so we have

$$
(1-\alpha) B+\alpha=\frac{(1-\alpha) A}{1-\alpha \rho}
$$

This expression can be rearranged into a quadratic in $\alpha$, which is guaranteed a solution by condition (6) in the text.

Let $\alpha^{*}$ be the solution. We need to find the fraction of clubbies in the population that will result in a steady state fraction of $\left(1-\alpha^{*}\right)$ clubbies entering the market every period. The total population size is normalized to 1 , and let $\beta$ be the number of clubbies in the population, with $\beta^{m}$ be the number in market and $\beta^{\circ}$ the number that are matched. Since parasites are always in the market, the total market population is $\left(1-\beta^{\circ}\right)$, and by definition:

$$
\beta^{m} /\left(1-\beta^{\circ}\right)=\left(1-\alpha^{*}\right)
$$

In equilibrium the outflow from the market must be equal to the inflow. The outflow is given by the number of long term matches which are formed each period, or $\left(\beta^{m}\right)^{2}$. The inflow is given by the number of long term matches that die at the end of the period, or $(1-\rho)\left(\left(\beta^{m}\right)^{2}+\beta^{o}\right)$. We therefore get the flow equation:

$$
\left(\beta^{m}\right)^{2}=(1-\rho)\left(\left(\beta^{m}\right)^{2}+\beta^{o}\right)
$$

we find that the equilibrium proportions are:

$$
\begin{aligned}
\beta^{m} & =\frac{-1+\sqrt{1+4 \rho\left(1-\alpha^{*}\right)^{2}}}{2 \rho\left(1-\alpha^{*}\right)}, \\
\beta^{o} & =\rho\left(\beta^{m}\right)^{2} .
\end{aligned}
$$

At this fraction of clubbies, new players will be indifferent between choosing to be a parasite or joining the group of clubbies. To complete the proof one needs to observe that no other strategy can do better against this profile. This would require that one be able to distinguish between the parasites and the clubbies. Since there are no reputation effects, and both types send the same messages, this is impossible. 
The computation of $\alpha^{*}$ is shown in Figure 2 .

Figure 2 here

From condition 6 in the text we see that a Nash equilibrium involving cooperation exists whenever the gains from cheating $(B-A)$ are sufficiently small and so long a $\rho$ is sufficiently large. Secondly, as one can see in the figure, when a Nash equilibrium exists there will typically be two of them, with the one having the smaller fraction of parasites being locally stable. 


\section{References}

[1] Akerlof, G., "Labor Contracts as a Partial Gift Exchange,"

Quarterly Journal of Economics 47 (1983), 543-69.

[2] Axelrod, R., The Evolution of Cooperation, (New York: Basic Books, 1984).

[3] Becker, G., A Treatise on the Family, (Cambridge: Harvard University Press, 1981).

[4] Binmore, K., and L. Samuelson, "Evolutionary Stability in Repeated Games Played by Finite Automata," Journal of Economic Theory 55 (1992), 278-305.

[5] Blume, A., Y. Kim, and J. Sobel, "Evolutionary Stability in Games of Communication," working paper, Department of Economics, University of California at San Diego, August 1992.

[6] Boas, F., Anthropology and Modern Life, (New York: W. W. Norton ,1928).

[7] Boyd, R., and P. Richerson, Culture and the Evolutionary Process, (Chicago: University of Chicago Press, 1985).

[8] Carmichael, H. L., "Firm Specific Human Capital and Promotion Ladders," Bell Journal 14 (1983), 251-58.

[9] Carmichael, H. L., "Self Enforcing Contracts, Shirking, and Life Cycle Incentives," Journal of Economic Perspectives 3 (1989), 65 - 83.

[10] Camerer, C., "Gifts as Economic Signals and Social Signals," American Journal of Sociology 94, (1988), S180 - S214.

[11] Coleman, J. S., "Social Capital in the Creation of Human Capital," American Journal of Sociology 94 (1988), S95 - S120.

[12] Dawkins, R., The Selfish Gene (Oxford: Oxford University Press, 1976) 
[13] Frank, R. H., "If Homo Economicus could Choose his own Utility Function, Would he Want One with a Conscience?," American Economic Review 78 (1988), 593-60.

[14] Fudenberg, D. J. and E. Maskin, "Evolution and Cooperation in Noisy Repeated Games," American Economic Review 80 (1990), 274-79.

[15] Maskin, E., "Evolution and Communication in Games," mimeo, Harvard University, 1993.

[16] Gauthier, D., Morals By Agreement (Cambridge: Cambridge University Press, 1986).

[17] Hofbauer, J. and K. Sigmund, The Theory of Evolution and Dynamical Systems, (Cambridge: Cambridge University Press, 1988).

[18] Kim, Y., and J. Sobel, "An Evolutionary Approach to Pre-Play Communications," working paper, University of Iowa, February 1992.

[19] Klein, B. and K. Leffler, "The Role of Market Forces in Ensuring Contractual Performance," Journal of Political Economy 89 (1979), 615-41.

[20] MacLeod, W. B. and J. Malcolmson, "Implicit Contracts, Incentive Compatability, and Involuntary Unemployment," Econometrica 57 (1989), 312-22.

[21] Matsui, A., "Cheap-Talk and Cooperation in a Society," Journal of Economic Theory 54 (1991), 245-258.

[22] Mauss, M. The Gift, (New York: W. W. Norton, 1990).

[23] Maynard Smith, J., Evolution and the Theory of Games (Cambridge: Cambridge University Press, 1982).

[24] Nowak, M. and K. Sigmund, "Tit for Tat in the Repeated Prisoner's Dilemma," Nature 355 (1992), 250-53.

[25] Robson, A., "Efficiency in Evolutionary Games: Darwin, Nash and the Secret Handshake," Journal of Theoretical Biology. 144 (1990), 379-96. 
[26] Shapiro, C. and J. Stiglitz, "Equilibrium Unemployment as a Worker Discipline Device," American Economic Review 74 (1984), 433-44.

[27] Schlag, K., "When Does Evolution Lead to Efficiency in Communication Games?," technical report, Friedrich-Wilhelms-Universitat Bonn, December1994.

[28] Taylor, P.D. and L. Jonker, "Evolutionarily Stable Strategies and Game Dynamics," Mathematical Biosciences 40 (1978), 145-56.

[29] Williamson, O., "Credible Commitments: the Use of Hostages to Support Exchange," American Economic Review 83 (1983), 519-40.

[30] van Damme, E., "Stable Equilibria and Forward Induction," Journal of Economic Theory 48 (1989), 476-98.

[31] van Damme, E., Stability and Perfection of Nash Equilibria (Berlin: Springer-Verlag, 1991).

\section{Figures}

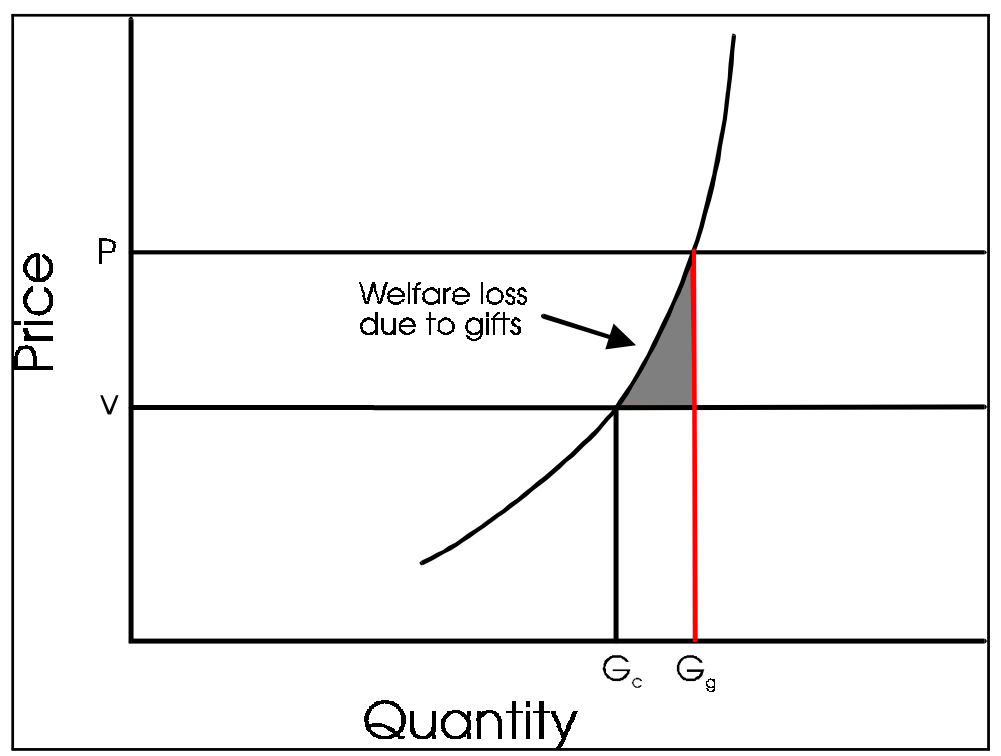

Figure 1: Figure 1: Welfare Loss due to Gifts 


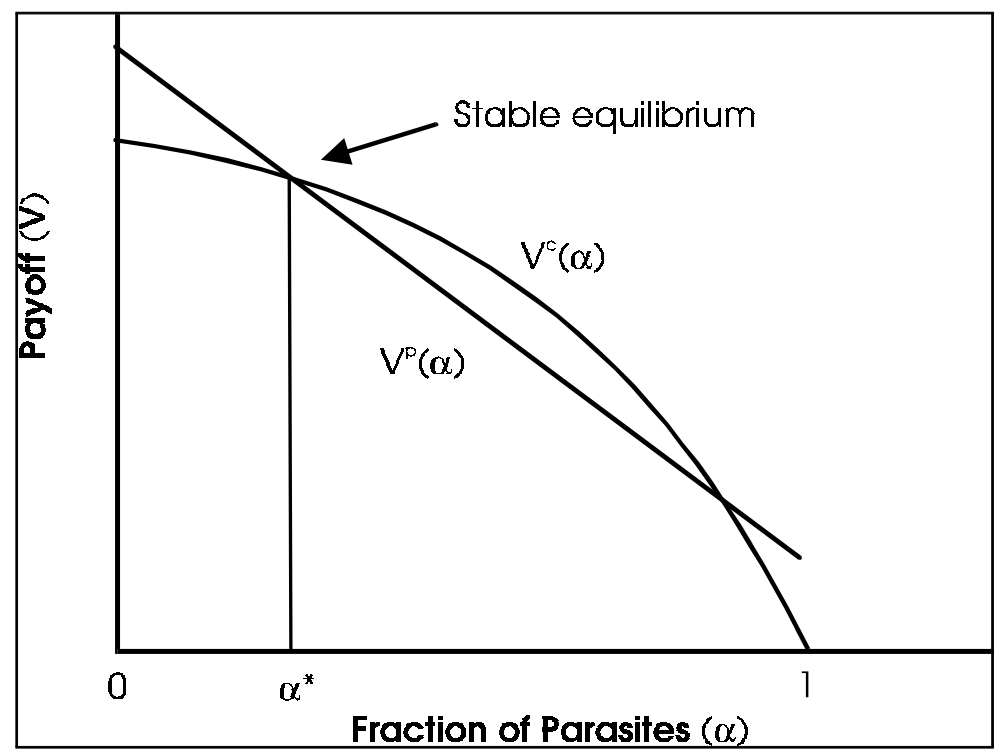

Figure 2: Figure 2: Equilibrium with Two Populations 